PROCEEDINGS OF THE

AMERICAN MATHEMATICAL SOCIETY

Volume 133, Number 5, Pages 1379-1386

S 0002-9939(04)07640-3

Article electronically published on October 18, 2004

\title{
MULTIPLIERS OF WEIGHTED SPACES AND REFLEXIVITY PROPERTY
}

\author{
XAVIER DUSSAU
}

(Communicated by Joseph A. Ball)

\begin{abstract}
We prove for some translation-invariant weighted spaces $E$ the following characterization: $T$ is a multiplier of $E$ if and only if $T$ leaves invariant every translation-invariant subspace of $E$. This result is equivalent with the reflexivity of the multiplier algebra of $E$.
\end{abstract}

\section{INTRODUCTION}

Let $G$ be a locally compact abelian group. If $x \in G$ and $f$ is a function on $G$, we define

$$
S_{x} f(t)=f(t-x), \forall t \in G .
$$

Let $E, F$ be two translation-invariant spaces of functions on $G$. A multiplier from $E$ to $F$ is a bounded operator $T$ from $E$ to $F$ which satisfies $S_{x} T=T S_{x}$ for every $x \in G$. The set of multipliers between $E$ and $F$ is denoted $(E, F)$. The multiplier problem is to characterize the multipliers between two spaces. For example, the Fourier-Plancherel Formula implies that $T \in\left(L^{2}(G), L^{2}(G)\right)$ if and only if there exists $\widehat{T} \in L^{\infty}(\Gamma)$, which satisfies $\widehat{T f}=\widehat{T} \widehat{f}$ for every $f \in L^{2}(G)$, where $\Gamma$ is the dual group of $G$. There exist a lot of results in Multiplier Theory (see [Tew96], Lar69]).

In this paper $\mathbb{C}, \mathbb{R}, \mathbb{Z}, \mathbb{D}$ and $\mathbb{T}$ denote respectively the complex field, the real field, the set of integers, the open unit disk and the unit circle.

A weight $\omega$ on $\mathbb{R}$ is a measurable function from $\mathbb{R}$ into $] 0,+\infty[$ such that

$$
\tilde{\omega}(x):=\sup _{t \in \mathbb{R}} \frac{\omega(t+x)}{\omega(t)}
$$

is bounded on every compact subset of $\mathbb{R}$. A weight $\omega$ is called dissymmetric if $\omega(t)=1$ for $t \geq 1$ and $\lim _{t \rightarrow-\infty} \omega(t)=+\infty$. We introduce the following Hilbert space:

$L_{\omega}^{2}(\mathbb{R}):=\left\{f: \mathbb{R} \rightarrow \mathbb{C}\right.$, measurable, $\left.\|f\|_{L_{\omega}^{2}(\mathbb{R})}=\left(\int_{-\infty}^{+\infty}|f(t) \omega(t)|^{2} d t\right)^{1 / 2}<+\infty\right\}$.

Received by the editors October 15, 2003 and, in revised form, January 2, 2004.

2000 Mathematics Subject Classification. Primary 47A15, 43A22; Secondary 46E25, 20C20.

Key words and phrases. Multiplier, translation invariant subspace, reflexivity.

(C)2004 American Mathematical Society Reverts to public domain 28 years from publication 
On this space, the scalar product is $[u, v]=\int_{-\infty}^{+\infty} f(t) \overline{g(t)} \omega(t)^{2} d t$. One can show that the translation operator $S_{x}$ is a bounded and invertible operator for every $x \in \mathbb{R}$. A subspace (i.e. closed linear subspace) $G$ of $L_{\omega}^{2}(\mathbb{R})$ is said to be translation invariant if $S_{x}(G) \subset G$ for $x \in \mathbb{R}$.

The algebra of bounded operators on a Hilbert space $H$ is denoted $\mathcal{B}(H)$. Let $\mathcal{E}$ be a subset of $\mathcal{B}(H)$. The lattice of invariant subspaces of $\mathcal{E}, \operatorname{Lat}(\mathcal{E})$, is the set of subspaces of $H$ which are invariant under every $T \in \mathcal{E}$. A subalgebra $\mathcal{U}$ of $\mathcal{B}(H)$ is reflexive when $\operatorname{Lat}(T) \supset \operatorname{Lat}(\mathcal{U})$ implies $T \in \mathcal{U}$. If $\mathcal{P} \subset \mathcal{B}(H)$, the commutant of $\mathcal{P}$ is called $\mathcal{P}^{\prime}$. An operator $T$ is hyperreflexive when $\{T\}^{\prime}$, the commutant of $T$, is a reflexive algebra. A subspace $F$ is said to be hyperinvariant for $T$ if $F \in \operatorname{Lat}\left(\{T\}^{\prime}\right)$. Donald Sarason ([Sar66]) introduced the reflexivity property and he proved that every normal operator is reflexive. See [CE00] or [Che92] for references about reflexivity results.

In this paper, we will prove the following theorem:

Theorem 1.1. If $\omega$ is a dissymmetric weight on $\mathbb{R}$, then $\left(L_{\omega}^{2}(\mathbb{R}), L_{\omega}^{2}(\mathbb{R})\right)$ is a reflexive algebra.

In 1981, Y. Domar Dom81 proved that for every weight $\omega$ of $\mathbb{R}$, there exist nontrivial translation invariant subspaces in $L_{\omega}^{2}(\mathbb{R})$. This result and Lemma 2.1 imply that $\operatorname{Lat}\left(L_{\omega}^{2}(\mathbb{R}), L_{\omega}^{2}(\mathbb{R})\right)$ is nontrivial for every weight $\omega$. Hence, it is interesting to know if $\left(L_{\omega}^{2}(\mathbb{R}), L_{\omega}^{2}(\mathbb{R})\right)$ enjoys the reflexivity property. This result is obvious if $\omega=1$, and we prove it in the dissymmetric case. The ingredients of our theorem are the estimates of [Dus02], Kapustin's reflexivity result Kap92], and some lemmas proved in section 2 .

Finally, Theorem 3.1 yields an abstract characterization of multipliers on $L_{\omega}^{2}(\mathbb{R})$. Indeed, we have the following corollary:

Corollary 1.2. If $\omega$ is a dissymmetric weight on $\mathbb{R}$, then $T \in\left(L_{\omega}^{2}(\mathbb{R}), L_{\omega}^{2}(\mathbb{R})\right)$ if and only if $T(F) \subset F$ for every translation invariant subspace $F$ of $L_{\omega}^{2}(\mathbb{R})$.

The author thanks Prof. Jean Esterle for his advice during the preparation of this paper.

\section{Some LEMmas}

A weight $\omega$ on $\mathbb{Z}$, is a map $\omega$ from $\mathbb{Z}$ into $] 0,+\infty[$ such that

$$
0<\inf _{n \in \mathbb{Z}} \frac{\omega(n+1)}{\omega(n)} \leq \sup _{n \in \mathbb{Z}} \frac{\omega(n+1)}{\omega(n)}<+\infty .
$$

Note that if $\omega$ is a weight on $\mathbb{R}$, then, $\omega_{\mid \mathbb{Z}}$ (still called $\omega$ ) is a weight on $\mathbb{Z}$. Let us introduce another Hilbert space:

$$
\ell_{\omega}^{2}(\mathbb{Z}):=\left\{u=\left(u_{n}\right)_{n \in \mathbb{Z}} \subset \mathbb{C} /\|u\|_{\ell_{\omega}^{2}(\mathbb{Z})}=\left[\sum_{n \in \mathbb{Z}}\left|u_{n}\right|^{2} \omega(n)^{2}\right]^{1 / 2}<\infty\right\} .
$$

A weight $\omega$ on $\mathbb{Z}$ is called dissymmetric if $\omega(n)=1$ for $n \geq 0$ and $\lim _{n \rightarrow-\infty} \omega(n)=$ $+\infty$.

If $\omega$ is a weight on $\mathbb{Z}$, then the bilateral weighted shift $S:\left(u_{n}\right)_{n \in \mathbb{Z}} \mapsto\left(u_{n-1}\right)_{n \in \mathbb{Z}}$ is a bounded and invertible operator of $\ell_{\omega}^{2}(\mathbb{Z})$. A subspace $F$ of $\ell_{\omega}^{2}(\mathbb{Z})$ is translation invariant if $S^{n}(F) \subset F$ for $n \in \mathbb{Z}$. In fact, $F$ is a translation invariant subspace of $\ell_{\omega}^{2}(\mathbb{Z})$ if and only if $F$ is a hyperinvariant subspace of $S$ (see [Shi74]). 
A translation invariant subspace $M$ of $\ell_{\omega}^{2}(\mathbb{Z})\left(\right.$ resp. $\left.L_{\omega}^{2}(\mathbb{R})\right)$ is said to be nontrivial (NT-TIS) if $M \neq\{0\}$ and $M \neq \ell_{\omega}^{2}(\mathbb{Z})$ (resp. $M \neq L_{\omega}^{2}(\mathbb{R})$ ). Note that the existence of a NT-TIS in $\ell_{\omega}^{2}(\mathbb{Z})$ is still unknown for all weights on $\mathbb{Z}$ (see Atz00] for references on this open problem). J. Esterle Est01 proved that Apostol weighted shifts are hyper-reflexive, and the author (Dus03], Dus02]) obtained also some hyperreflexivity results.

Given a weight $\omega$ on $\mathbb{R}$ we introduce the weight $\omega^{*}(t):=\frac{1}{\omega(-t)}$.

We have $\left(L_{\omega}^{2}(\mathbb{R})\right)^{*}=L_{\omega^{*}}^{2}(\mathbb{R})$ with the following duality:

$$
\langle f, g\rangle=\int_{\mathbb{R}} f(t) g(-t) d t, f \in L_{\omega}^{2}(\mathbb{R}), g \in L_{\omega^{*}}^{2}(\mathbb{R}) .
$$

The following result is probably known, but we did not see it in the literature.

Lemma 2.1. Let $\omega$ be a weight on $\mathbb{R}$; then Lat $\left(L_{\omega}^{2}(\mathbb{R}), L_{\omega}^{2}(\mathbb{R})\right)=\operatorname{Lat}\left(\left\{S_{t}: t \in \mathbb{R}\right\}\right)$.

Proof. Clearly $\operatorname{Lat}\left(L_{\omega}^{2}(\mathbb{R}), L_{\omega}^{2}(\mathbb{R})\right)$ is included in the set of translation invariant subspaces of $L_{\omega}^{2}(\mathbb{R})$. Let $u, v \in L_{\omega}^{2}(\mathbb{R})$ with $\left[S_{-t} u, v\right]=0$ for $t \in \mathbb{R}$, and let $R \in\left(L_{\omega}^{2}(\mathbb{R}), L_{\omega}^{2}(\mathbb{R})\right)$. Define $f(t)=\left[S_{-t} R u, v\right]$ for $t \in \mathbb{R}$. Let $u \in \mathcal{D}(\mathbb{R})$, the space of infinitely differentiable functions with compact support. Then the Lebesgue convergence theorem yields $\lim _{y \rightarrow x}\left\|S_{y} u-S_{x} u\right\|_{\omega}=0$ for $x \in \mathbb{R}$. Clearly, $\mathcal{D}(\mathbb{R})$ is a dense subspace of $L_{\omega}^{2}(\mathbb{R})$. Thus the map $x \mapsto S_{x} u$ from $\mathbb{R}$ into $L_{\omega}^{2}(\mathbb{R})$ is continuous for every $u \in L_{\omega}^{2}(\mathbb{R})$. Hence $f$ is continuous on the line. We will prove that $f$ is the zero distribution.

Let $k \in \mathcal{D}(\mathbb{R})$, and $\tilde{k}(t):=k(-t)$. Notice that $\tilde{k} \in L_{\omega^{*}}^{2}(\mathbb{R})$. We obtain

$$
\begin{aligned}
\int_{\mathbb{R}} f(y) k(y) d y & =\int_{\mathbb{R}}\left[\int_{\mathbb{R}}(R u)(x+y) \overline{v(x)} \omega(x)^{2} d x\right] k(y) d y \\
& =\int_{\mathbb{R}}\left[\int_{\mathbb{R}}(R u)(y+x) k(y) d y\right] \overline{v(x)} \omega(x)^{2} d x \\
& =\int_{\mathbb{R}}\left\langle S_{-x} R u, \tilde{k}\right\rangle \overline{v(x)} \omega(x)^{2} d x \\
& =\int_{\mathbb{R}}\left\langle R S_{-x} u, \tilde{k}\right\rangle \overline{v(x)} \omega(x)^{2} d x \\
& =\int_{\mathbb{R}}\left\langle S_{-x} u,{ }^{t} R \tilde{k}\right\rangle \overline{v(x)} \omega(x)^{2} d x \\
& =\int_{\mathbb{R}}\left[S_{-y} u, v\right]\left({ }^{t} R \tilde{k}\right)(-y) d y \\
& =0
\end{aligned}
$$

where ${ }^{t} R \in \mathcal{B}\left(L_{\omega^{*}}^{2}(\mathbb{R})\right)$ denotes the adjoint operator of $R$. Thus $f=0$ a.e. The continuity of $f$ implies $f(0)=0$. Therefore, if $\left[S_{-t} u, v\right]=0$ for $t \in \mathbb{R}$, then we have $[R u, v]=0$. Thus,

$$
R u \in \operatorname{span}\left(S_{-t} u, t \in \mathbb{R}\right),
$$

for $u \in L_{\omega}^{2}(\mathbb{R})$, where $\operatorname{span}(A)$ denotes the closed linear subspace generated by $A$. From this, it easily follows that every translation invariant subspace of $L_{\omega}^{2}(\mathbb{R})$ belongs to $\operatorname{Lat}\left(L_{\omega}^{2}(\mathbb{R}), L_{\omega}^{2}(\mathbb{R})\right)$.

Remark 2.2. Let $\omega$ be a weight on $\mathbb{Z}$. It is known that $\operatorname{Lat}\left(\left\{S^{n}: n \in \mathbb{Z}\right\}^{\prime}\right)=$ Lat $\left\{S^{n}: n \in \mathbb{Z}\right\}$. Indeed, $\left(\ell_{\omega}^{2}(\mathbb{Z}), \ell_{\omega}^{2}(\mathbb{Z})\right)$ is the closed algebra, under the strong 
operator topology, generated by $S$ and $S^{-1}$ (see [Shi74]). A similar result is still unknown for multipliers of $L_{\omega}^{2}(\mathbb{R})$.

The dual of $\ell_{\omega}^{2}(\mathbb{Z})$ could be identified to $\ell_{\omega^{*}}^{2}(\mathbb{Z})$ with the following duality:

$$
\langle u, v\rangle=\sum_{n \in \mathbb{Z}} u_{n} v_{-n}, u \in \ell_{\omega}^{2}(\mathbb{Z}), v \in \ell_{\omega^{*}}^{2}(\mathbb{Z}) .
$$

If $F$ is an NT-TIS of $\ell_{\omega}^{2}(\mathbb{Z}), F^{\perp}$ will denote the orthogonal complement of $F$ in the preceding duality. We have

$$
\left(\ell_{\omega}^{2}(\mathbb{Z}) / F\right)^{*}=F^{\perp}
$$

with the following bilinear map: $(P(u), v) \mapsto\langle u, v\rangle$, where $P$ is the natural map from $\ell_{\omega}^{2}(\mathbb{Z})$ into $\ell_{\omega}^{2}(\mathbb{Z}) / F$.

For $n \in \mathbb{Z}, e_{n}$ is the sequence which satisfies $e_{n}(n)=1$ and $e_{n}(k)=0$ for $k \neq n$. Denote by $\log (z):=\log |z|+i \arg _{0,2 \pi[}(z)$ the logarithm branch in $\mathbb{C} \backslash[0,+\infty[$.

Lemma 2.3. Let $\omega$ be a weight on $\mathbb{R}$ and $F$ an NT-TIS of $\ell_{\omega}^{2}(\mathbb{Z})$ such that the spectrum of the operator $\tilde{S}: u+F \rightarrow S(u)+F$ is a subset of $\mathbb{C} \backslash[0,+\infty[$; then there exists a constant $C>0$ satisfying for every $g \in F^{\perp}$,

$$
\int_{-\infty}^{+\infty}\left|\left\langle e^{-t \log \tilde{S}} P\left(e_{0}\right), g\right\rangle\right|^{2} \frac{d t}{\omega(-t)^{2}} \leq C \sum_{n \in \mathbb{Z}} \frac{|g(n)|^{2}}{\omega(-n)^{2}} .
$$

Proof. Let $u(x)=\left\langle e^{-x \log \tilde{S}} P\left(e_{0}\right), g\right\rangle, x \in \mathbb{R}$. Given $n \in \mathbb{Z}$, denote by $u_{n}$ the map $u_{-n}(x)=u(x+n), x \in \mathbb{R}$. The Parseval formula in the Hilbert space $L^{2}([0,1])$ yields

$$
\int_{n}^{n+1}|u(t)|^{2} d t=\int_{0}^{1}\left|u_{-n}(t)\right|^{2} d t=\sum_{p \in \mathbb{Z}}\left|\widehat{u_{-n}}(p)\right|^{2} .
$$

The spectrum of $\log \tilde{S}$ is included in $\{z \in \mathbb{C}: \operatorname{Im}(z) \in] 0,2 \pi[\}$; thus we can define for each $p \in \mathbb{Z}$ the operator $A_{p}=(\log \tilde{S}+2 i p \pi I)^{-1}\left(I-\tilde{S}^{-1}\right) \in \mathcal{B}\left(\ell_{\omega}^{2}(\mathbb{Z}) / F\right)$. It is fairly easy to prove that $\widehat{u_{-n}}(p)=\left\langle A_{p} P\left(e_{-n}\right), g\right\rangle$. Hence,

$$
\int_{0}^{1}\left|u_{-n}(t)\right|^{2} d t=\sum_{p \in \mathbb{Z}}\left|\left\langle A_{p} P\left(e_{-n}\right), g\right\rangle\right|^{2}=\sum_{p \in \mathbb{Z}}\left|\left\langle P\left(e_{-n}\right),{ }^{t} A_{p} g\right\rangle\right|^{2} .
$$

Letting $C=\sup _{x \in[0,1]} \tilde{\omega}(x)^{2}$, we obtain

$$
\begin{aligned}
\int_{-\infty}^{+\infty}|u(t)|^{2} \frac{d t}{\omega(-t)^{2}} & \leq C \sum_{n \in \mathbb{Z}} \frac{1}{\omega(-n)^{2}} \int_{n}^{n+1}|u(t)|^{2} d t \\
& =C \sum_{n \in \mathbb{Z}} \frac{1}{\omega(-n)^{2}} \sum_{p \in \mathbb{Z}}\left|\left\langle P\left(e_{-n}\right),{ }^{t} A_{p} g\right\rangle\right|^{2} \\
& =C \sum_{p \in \mathbb{Z}} \sum_{n \in \mathbb{Z}} \frac{\left|\left\langle P\left(e_{-n}\right),{ }^{t} A_{p} g\right\rangle\right|^{2}}{\omega(-n)^{2}} \\
& =C \sum_{p \in \mathbb{Z}}\left\|{ }^{t} A_{p} g\right\|_{\ell_{\omega^{*}}^{2}(\mathbb{Z})}^{2} \\
& \leq C \sum_{p \in \mathbb{Z}}\left\|^{t} A_{p}\right\|^{2}\|g\|_{\ell_{\omega^{*}}^{2}(\mathbb{Z})}^{2} .
\end{aligned}
$$

Clearly $\left\|A_{p}\right\|=O\left(\frac{1}{|p|}\right)$ if $|p| \rightarrow+\infty$; thus $\sum_{p \in \mathbb{Z}}||^{t} A_{p} \|^{2}<+\infty$. 
Several authors obtained similar estimations in Banach algebras, which are not usable here. By chance, we obtained this lemma with an unexpected use of the Parseval Formula. The following lemma is a link between the discrete and the continuous case, and it is interesting by itself.

Lemma 2.4. Let $\omega$ be a weight on $\mathbb{R}$ and $F$ an $N T$-TIS of $\ell_{\omega}^{2}(\mathbb{Z})$ such that the spectrum of the operator $\tilde{S}: u+F \rightarrow S(u)+F$ is a subset of $\mathbb{C} \backslash[0,+\infty[$; then there exists an NT-TIS G of $L_{\omega}^{2}(\mathbb{R})$ such that $\tilde{S}$ is similar to $\tilde{S}_{1}: f+G \rightarrow S_{1}(f)+G$.

Proof. The set of step functions on the line will be denoted $\mathcal{E}$. Let

$$
T f=\int_{-\infty}^{+\infty} f(t) e^{t \log \tilde{S}} P\left(e_{0}\right) d t,
$$

which is a linear map from $\mathcal{E} \subset L_{\omega}^{2}(\mathbb{R})$ to $\ell_{\omega}^{2}(\mathbb{Z}) / F$. Let $f \in \mathcal{E}, g \in F^{\perp}$; we obtain

$$
\begin{aligned}
|\langle T f, g\rangle| & =\left|\int_{\mathbb{R}} f(t)\left\langle e^{t \log \tilde{S}} P\left(e_{0}\right), g\right\rangle d t\right| \\
& \leq\left(\int_{\mathbb{R}}|f(t)|^{2} \omega(t)^{2} d t\right)^{1 / 2}\left(\int_{\mathbb{R}}\left|\left\langle e^{-t \log \tilde{S}} P\left(e_{0}\right), g\right\rangle\right|^{2} \frac{d t}{\omega(-t)^{2}}\right)^{1 / 2} .
\end{aligned}
$$

The preceding lemma implies the existence of a constant $C$ such that

$$
|\langle T f, g\rangle| \leq C|| f\left\|_{L_{\omega}^{2}(\mathbb{R})} \mid\right\| g \|_{\ell_{\omega^{*}}^{2}(\mathbb{Z})} .
$$

Thus, $\|T f\| \leq C\|f\|_{L_{\omega}^{2}(\mathbb{R})}$ for every $f \in \mathcal{E}$. The closure of $\mathcal{E}$ is equal to $L_{\omega}^{2}(\mathbb{R})$; hence $T$ is a bounded operator from $L_{\omega}^{2}(\mathbb{R})$ into $\ell_{\omega}^{2}(\mathbb{Z}) / F$. We will prove that $T$ is surjective. The characteristic function of $A \subset \mathbb{R}$ is denoted $\chi_{A}$. Let $B=$ $\left\{\sum_{n \in \mathbb{Z}} u_{n} \chi_{[n, n+1[}: u=\left(u_{n}\right)_{n} \in \ell_{\omega}^{2}(\mathbb{Z})\right\}$. Clearly, $B$ is a subspace of $L_{\omega}^{2}(\mathbb{R})$ and the map $J(u)=\sum_{n \in \mathbb{Z}} u_{n} \chi_{[n, n+1[}$ is a bounded invertible operator from $\ell_{\omega}^{2}(\mathbb{Z})$ into $B$. Note that $J\left(e_{n}\right)=\chi_{[n, n+1[}$. Let $R:=\int_{0}^{1} e^{t \log \tilde{S}} d t$, which is an invertible operator of $\ell_{\omega}^{2}(\mathbb{Z}) / F$ because $R=(\log \tilde{S})^{-1}(\tilde{S}-I)$. Let $P$ be the natural map from $\ell_{\omega}^{2}(\mathbb{Z})$ into $\ell_{\omega}^{2}(\mathbb{Z}) / F$ and $n \in \mathbb{Z}$. We have

$$
\begin{aligned}
R P\left(e_{n}\right) & =R\left(\tilde{S}^{n} P\left(e_{0}\right)\right) \\
& =\int_{0}^{1} e^{t \log \tilde{S} \tilde{S}^{n} P\left(e_{0}\right) d t} \\
& =\int_{n}^{n+1} e^{t \log \tilde{S}} P\left(e_{0}\right) d t \\
& =\int_{-\infty}^{+\infty} \chi_{[n, n+1[}(t) e^{t \log \tilde{S}} P\left(e_{0}\right) d t \\
& =T J\left(e_{n}\right) .
\end{aligned}
$$

Hence, $T J=R P$. The operator $R P$ is surjective; therefore $T$ is surjective. One can show that

$$
T S_{x}=e^{x \log \tilde{S}} T \forall x \in \mathbb{R} .
$$

Hence, $\operatorname{Ker}(T)$ is a translation invariant subspace of $L_{\omega}^{2}(\mathbb{R})$. We have $\operatorname{Ker} T \supset J(F)$ and $\operatorname{Ker} T \neq L_{\omega}^{2}(\mathbb{R})$ because $T$ is surjective. Hence, $\operatorname{Ker}(T)$ is an NT-TIS of $L_{\omega}^{2}(\mathbb{R})$. Let the operator $\tilde{T}: L_{\omega}^{2}(\mathbb{R}) / \operatorname{Ker} T \rightarrow \ell_{\omega}^{2}(\mathbb{Z}) / F$ be induced by $T$. The operator $\tilde{T}$ is bounded and invertible, and relation 2.1 yields $\tilde{T} \tilde{S}_{1}=\tilde{S} \tilde{T}$. Therefore $\tilde{S}$ and $\tilde{S}_{1}$ are similar. 


\section{Multipliers of $L_{\omega}^{2}(\mathbb{R})$ With $\omega$ Dissymmetric}

Define a dissymmetric weight $\omega$ on $\mathbb{R}$ by $\omega(t)=1$ for $t \geq 0$ and $\lim _{t \rightarrow-\infty} \omega(t)=$ $+\infty$. The space $\left\{f \in \ell_{\omega}^{2}(\mathbb{Z}): f_{n}=0\right.$ for $\left.n<0\right\}$ can be identified with $H^{2}(\mathbb{D})$, the classical Hardy space. Let $U$ be a singular inner function, i.e. an inner function which does not vanish on $\mathbb{D}$. Then there exists a singular positive measure $\mu$ on $\mathbb{T}$ such that

$$
U_{\mu}(z)=\exp \left[-\int_{\mathbb{T}} \frac{\xi+z}{\xi-z} d \mu(\xi)\right] \text { for } z \in \mathbb{D} .
$$

Let $E(\widehat{U})$ be the translation invariant subspace of $\ell_{\omega}^{2}(\mathbb{Z})$ generated by $\widehat{U}$. We recall that a subset $A$ of the unit circle is a Carleson set if $m(A)=0$ and

$$
\sum_{k} \ell_{k} \log \left(\frac{2 \pi}{\ell_{k}}\right)<+\infty
$$

where $m$ is the Haar measure on $\mathbb{T}$ and $\ell_{k}$ are the lengths of the complementary $\operatorname{arcs}$ of $A$.

Theorem 3.1. If $\omega$ is a dissymmetric weight on $\mathbb{R}$, then $\left(L_{\omega}^{2}(\mathbb{R}), L_{\omega}^{2}(\mathbb{R})\right)$ is a reflexive algebra.

Proof. The author proved (see Dus02]) that if $\omega$ is dissymmetric, there exists a singular measure $\mu$, which vanishes on Carleson sets, such that the operator

$$
\tilde{S}: u+E\left(\widehat{U_{\mu}}\right) \mapsto S u+E\left(\widehat{U_{\mu}}\right)
$$

of $\ell_{\omega}^{2}(\mathbb{Z}) / E\left(\widehat{U_{\mu}}\right)$ is similar to

$$
M_{U}: f+U_{\mu} H^{2}(\mathbb{D}) \mapsto S f+U_{\mu} H^{2}(\mathbb{D}) .
$$

Of course, $\mu$ is the measure associated with $U$. So, $M_{U}$ is a bounded operator of $H^{2}(\mathbb{D}) / U_{\mu} H^{2}(\mathbb{D})$.

V.V. Kapustin Kap92 proved that the operator $M_{U}$ is hyper-reflexive if and only if the measure $\mu$ vanishes on Carleson sets. Hence the operator $\tilde{S}$ is hyperreflexive. The measure $\mu$ was obtained by lemma 5.6 of [Est97]. Moreover, it is proved in this lemma that the supporting set of $\mu$ is a perfect set of zero measure. Changing $\mu$ into $\alpha \mu$, where $|\alpha|=1$, we can suppose without loss of generality that $\operatorname{supp}(\mu) \subset \mathbb{T} \backslash\{1\}$. Here, $\alpha \mu$ denotes the image measure of $\mu$ with the map $z \rightarrow \alpha z$. We have $\sigma\left(M_{U}\right)=\operatorname{supp}(\mu)($ SNF67, p. 117]); hence $\sigma(\tilde{S})=\operatorname{supp}(\mu) \subset \mathbb{T} \backslash\{1\}$.

Therefore it is possible to use Lemma 2.4: there exists an NT-TIS $G$ of $L_{\omega}^{2}(\mathbb{R})$ such that the shift $\tilde{S}_{1}$ on $L_{\omega}^{2}(\mathbb{R}) / G$ is similar to the operator $\tilde{S}$ on $\ell_{\omega}^{2}(\mathbb{Z}) / E(\widehat{U})$. The operator $\tilde{S}$ is hyper-reflexive, hence $\tilde{S}_{1}$ is hyper-reflexive.

Let $x_{0} \in \mathbb{R}$, and let $R$ be a bounded operator on $L_{\omega}^{2}(\mathbb{R})$ which satisfies $\operatorname{Lat}(R) \supset$ $\operatorname{Lat}\left(L_{\omega}^{2}(\mathbb{R}), L_{\omega}^{2}(\mathbb{R})\right)$. Lemma 2.1 implies that $\operatorname{Lat}(R) \supset \operatorname{Lat}\left\{S_{t} ; t \in \mathbb{R}\right\}$. Let $\tilde{R}: f+G \rightarrow R f+G$. It is easy to prove that $\tilde{R}$ leaves invariant every hyperinvariant subspace of $\tilde{S}_{1}$. The operator $\tilde{S}_{1}$ is hyper-reflexive; hence $\tilde{R} \tilde{S}_{1}=\tilde{S}_{1} \tilde{R}$. Clearly, we have also $\tilde{S_{x_{0}}} \tilde{S_{1}}=\tilde{S_{1}} \tilde{S_{x_{0}}}$, where $\tilde{S_{x_{0}}}(f+G)=S_{x_{0}} f+G$. It is a standard result (see [SNF67]) that the commutant of $M_{U}$ equals the commutative algebra $H^{\infty}(\mathbb{D}) / U H^{\infty}(\mathbb{D})$, where $H^{\infty}(\mathbb{D})$ denotes the space of bounded holomorphic functions on the disk $\mathbb{D}$. Hence, the commutant of $\tilde{S}_{1}$ is a commutative algebra because $\tilde{S_{1}}$ and $M_{U}$ are similar. So, $\tilde{S_{x_{0}}} \tilde{R}=\tilde{R} \tilde{S_{x_{0}}}$ and this implies that $S_{x_{0}} R f-R S_{x_{0}} f \in G$ for $f \in L_{\omega}^{2}(\mathbb{R})$. 
For each $\theta \in \mathbb{R}$, let $G_{\theta}=\left\{f \in L_{\omega}^{2}(\mathbb{R}): t \mapsto f(t) e^{i t \theta} \in G\right\}$. Let $K_{\theta}$ be the linear map from $L_{\omega}^{2}(\mathbb{R})$ into itself which satisfies $K_{\theta}(f)(t)=f(t) e^{i t \theta}$. It is easy to prove that $K_{\theta}$ is a surjective isometry of $L_{\omega}^{2}(\mathbb{R}), K_{\theta}\left(G_{\theta}\right)=G$, and that $G_{\theta}$ is an NT-TIS of $L_{\omega}^{2}(\mathbb{R})$. Let $\tilde{K}_{\theta}: f+G_{\theta} \rightarrow f+G$, the operator from $L_{\omega}^{2}(\mathbb{R}) / G_{\theta}$ into $L_{\omega}^{2}(\mathbb{R}) / G$ induced by $K_{\theta}$ and $\tilde{T}_{1}\left(f+G_{\theta}\right)=S_{1} f+G_{\theta}$, the shift operator on $L_{\omega}^{2}(\mathbb{R}) / G_{\theta}$. We have $\tilde{K}_{\theta} \tilde{T}_{1}=\exp (i \theta) \tilde{S}_{1} \tilde{K}_{\theta}$; hence $\exp (-i \theta) \tilde{T}_{1}$ is similar to $\tilde{S}_{1}$. Let $R$ be an operator on $L_{\omega}^{2}(\mathbb{R})$ which leaves invariant every translation invariant subspace, and let $\tilde{R}$ : $f+G_{\theta} \rightarrow R f+G_{\theta}$. It is easy to prove that $\tilde{R}$ leaves invariant every hyperinvariant subspace of $\exp (-i \theta) \tilde{T}_{1}$. The operator $\tilde{S}_{1}$ is hyper-reflexive; hence $\exp (-i \theta) \tilde{T}_{1}$ is also hyper-reflexive. Therefore, $\tilde{R} \tilde{T}_{1}=\tilde{T}_{1} \tilde{R}$. Using the same argument as above, we obtain $S_{x_{0}} R f-R S_{x_{0}} f \in G_{\theta}$, for every $f \in L_{\omega}^{2}(\mathbb{R})$. Our arguments are valid for each $\theta \in \mathbb{R}$. So we have proved

$$
S_{x_{0}} R f-R S_{x_{0}} f \in \bigcap_{\theta \in \mathbb{R}} G_{\theta},
$$

for $f \in L_{\omega}^{2}(\mathbb{R})$.

The subspace $G$ is an NT-TIS; hence there exists $g \in L_{\omega}^{2}(\mathbb{R}), \neq 0$ orthogonal with $G$. Therefore, there exists a bounded Borel set $A \subset \mathbb{R}$, with $m(A)>0$ and $g(t) \neq 0$ for $t \in A$. Suppose $\bigcap_{\theta \in \mathbb{R}} G_{\theta} \neq\{0\}$, and let a function $f \neq 0$ belong to this intersection; then there exists a bounded Borel set $B$, with $m(B)>0$ and $f(t) \neq 0$ for $t \in B$. If there does not exist $t \in \mathbb{R}$ satisfying $m\left(A \cap\left(B-t_{0}\right)\right)>0$, we would obtain $\widehat{\chi_{-A}}(t) \widehat{\chi_{B}}(t)=0$ for $t \in \mathbb{R}$; but this is impossible because $\widehat{\chi_{-A}}$ and $\widehat{\chi_{B}}$ are nonzero entire functions. Thus, there exists $t_{0} \in \mathbb{R}$ such that $m\left(A \cap\left(B-t_{0}\right)\right)>$ 0 . Moreover, $S_{t_{0}} f \in \bigcap_{\theta \in \mathbb{R}} G_{\theta}$, because this intersection is translation invariant. Therefore,

$$
\int_{-\infty}^{+\infty} f\left(t-t_{0}\right) e^{i t \theta} \overline{g(t)} \omega(t)^{2} d t=0, \forall \theta \in \mathbb{R}
$$

Hence, $f\left(t-t_{0}\right) \overline{g(t)}=0$ a.e.; and this is impossible because $f\left(t-t_{0}\right) \overline{g(t)} \neq 0$ for $t \in A \cap\left(B-t_{0}\right)$. So we obtain

$$
\bigcap_{\theta \in \mathbb{R}} G_{\theta}=\{0\},
$$

and $R S_{x_{0}}=S_{x_{0}} R$. The real $x_{0}$ is arbitrary; thus $R \in\left(L_{\omega}^{2}(\mathbb{R}), L_{\omega}^{2}(\mathbb{R})\right)$.

Corollary 3.2. If $\omega$ is a dissymmetric weight on $\mathbb{R}$, then $T \in\left(L_{\omega}^{2}(\mathbb{R}), L_{\omega}^{2}(\mathbb{R})\right)$ if and only if $T(F) \subset F$ for every translation invariant subspace $F$ of $L_{\omega}^{2}(\mathbb{R})$.

Proof. It is an obvious consequence of Lemma 2.1 and Theorem 1.1.

Note that this characterization is also true for $\left(\ell_{\omega}^{2}(\mathbb{Z}), \ell_{\omega}^{2}(\mathbb{Z})\right)$ when the bilateral weighted shift $S$ on $\ell_{\omega}^{2}(\mathbb{Z})$ is hyper-reflexive. Therefore, we have this characterization for all weighted spaces considered in [Est01, Dus03, Dus02].

\section{REFERENCES}

[Atz00] Aharon Atzmon, The existence of translation invariant subspaces of symmetric selfadjoint sequence spaces on Z, J. Funct. Anal. 178 (2000), no. 2, 372-380. MR 1802899 (2002a:46023)

[CE00] Isabelle Chalendar and Jean Esterle, Le problème du sous-espace invariant, Development of Mathematics 1950-2000, Birkhäuser, Basel, 2000, pp. 235-267. MR 1796843 (2001i:47005) 
[Che92] B. Chevreau, A survey of recent reflexivity results, Operator algebras and operator theory (Craiova, 1989), Pitman Res. Notes Math. Ser., vol. 271, Longman Sci. Tech., Harlow, 1992, pp. 46-61. MR 1189165 (93i:47066)

[Dom81] Yngve Domar, Translation invariant subspaces of weighted $l^{p}$ and $L^{p}$ spaces, Math. Scand. 49 (1981), no. 1, 133-144. MR0645094 (83k:47022)

[Dus02] Xavier Dussau, Les shifts à poids dissymétriques sont hyper-réflexifs, Bull. Soc. Math. France 130 (2002), no. 4, 573-585. MR1947453 (2003k:47039)

[Dus03] _ Les shifts à poids de croissance polynomiale sont hyper-réflexifs, Arch. Math. (Basel) 80 (2003), no. 1, 71-78. MR.1968289 (2003m:47060)

[Est97] Jean Esterle, Singular inner functions and biinvariant subspaces for dissymmetric weighted shifts, J. Funct. Anal. 144 (1997), no. 1, 64-104. MR1430716 (98d:47021)

[Est01] — Apostol's bilateral weighted shifts are hyper-reflexive, Recent advances in operator theory and related topics (Szeged, 1999), Oper. Theory Adv. Appl., vol. 127, Birkhäuser, Basel, 2001, pp. 243-266. MR 1902804 (2003c:47009)

[Kap92] V. V. Kapustin, Reflexivity of operators: general methods and a criterion for almost isometric contractions, Algebra i Analiz 4 (1992), no. 2, 141-160. MR.1182398 (93j:47028)

[Lar69] Ronald Larsen, The multiplier problem, Springer-Verlag, Berlin, 1969, Lecture Notes in Mathematics, Vol. 105. MR0435737|(55:8694)

[Sar66] D. Sarason, Invariant subspaces and unstarred operator algebras, Pacific J. Math. 17 (1966), 511-517.

[Shi74] Allen L. Shields, Weighted shift operators and analytic function theory, Topics in operator theory, Amer. Math. Soc., Providence, R.I., 1974, pp. 49-128. Math. Surveys, No. 13. MR.0361899 (50:14341)

[SNF67] Béla Sz.-Nagy and Ciprian Foias, Analyse harmonique des opérateurs de l'espace de Hilbert, Masson et Cie, Paris, 1967. MR0225183 (37:778)

[Tew96] U. B. Tewari, The multiplier problem, Math. Student 65 (1996), no. 1-4, 45-56. MR.1618307 (99d:43005)

Laboratoire de Mathématiques Pures, Université Bordeaux I, 351, COURS de la libération, 33405 Talence Cedex, France

E-mail address: dussau@math.u-bordeaux.fr 